\title{
DDS: A Delay-Constrained Duty-Cycle Scheduling Algorithm in Wireless Sensor Networks
}

\author{
Duy-Son Vu ${ }^{\circledR}$, Thi-Nga Dao ${ }^{\circledR}$ and Seokhoon Yoon * ${ }^{(1)}$ \\ Department of Electrical and Computer Engineering, University of Ulsan, Ulsan 680-749, Korea; \\ sonduyvu@gmail.com (D.-S.V.); daothinga.mta@gmail.com (T.-N.D.) \\ * Correspondence: seokhoonyoon@ulsan.ac.kr; Tel.: +82-52-259-1403
}

Received: 9 October 2018; Accepted: 3 November 2018; Published: 7 November 2018

check for updates

\begin{abstract}
Since sensor nodes usually have a large duty cycle interval to prolong network lifetime, duty-cycled wireless sensor networks (WSNs) can suffer from a long end-to-end (E2E) delay. Because delay-sensitive applications have a certain E2E delay requirement, a lot of studies have tried to tackle the long E2E delay problem. However, most existing studies focused on simply reducing the E2E delay rather than considering the delay bound requirement, which makes it hard to achieve balanced performance between E2E delay and energy efficiency. Although a few studies took into consideration both the delay bound requirement and energy consumption, they required specific node deployment or strict time synchronization between nodes in the network. In order to address the limitations of the existing studies, we propose a delay-constrained duty-cycle scheduling (DDS) algorithm. The objective of DDS is to achieve low energy consumption while satisfying the delay bound requirement in various node deployment scenarios depending on user demands. First, based on network topology information collected by the sink, one-hop delay distribution is derived as a function of the duty cycle interval. Then, the E2E delay distribution is estimated using the Lyapunov central limit theorem, which allows each node group to have a different delay distribution. Finally, the duty cycle interval is determined using the estimated E2E delay distribution such that energy consumption is minimized while meeting the delay bound requirement. Practical WSN deployment scenarios are considered to evaluate the proposed algorithm. The simulation results show that DDS can guarantee the given delay bound requirement and outperform existing algorithms in terms of energy efficiency.
\end{abstract}

Keywords: wireless sensor networks; duty cycle interval; delay distribution; delay requirement; delay bound

\section{Introduction}

Wireless sensor networks (WSNs) are widely used in various fields for practical applications, such as environmental monitoring, vehicle tracking, surveillance, and healthcare [1]. In WSNs, a large number of sensor nodes are deployed in a region to collect information, e.g., tracking and identifying the location of miners and roof falls in underground mine environments, or detecting a vehicle accident using monitoring cameras in vehicles $[2,3]$.

Since the primary power source of sensor nodes is a battery with limited capacity, reducing the power consumption to prolong network lifetime is a crucial challenge in WSNs. One of the most effective solutions for saving energy is the duty cycling mechanism [4,5] in which a node's radio is switched on for an active period and off for the rest of the duty cycle interval. Because sensor nodes only occasionally transmit and receive data packets in practice, having the sensor's radio continuously turned on is not necessary. 
Because sensor nodes consume low energy with a large duty cycle interval, a long network lifetime can be achieved by maximizing the duty cycle interval. However, a large duty cycle interval also leads to a long end-to-end (E2E) delay [6], which is the latency from packet generation time at the source node to reception time at the sink. As the duty cycle interval increases, nodes wake up less frequently. Therefore, the transmitters' waiting time for potential forwarding candidates to wake up becomes longer, which results in a longer E2E delay.

From those two aspects of the duty cycling mechanism, consideration of a balanced performance between energy consumption and E2E delay is desired, since a lot of applications in WSNs require a certain E2E delay bound, as well as low energy consumption. For example, in a fire warning system, because a long E2E delay can have serious consequences, packets may be required to reach the sink within a given delay bound at a certain probability.

Although a lot of studies [7-19] attempted to address long E2E delays in WSNs, they focused on simply reducing E2E delays, instead of taking the delay bound requirement into account. As a result, it is hard for them to achieve a balanced performance between E2E delay and energy consumption. We also note that a few studies [20-22] considered both the delay bound requirement and energy consumption. However, those works required additional information or certain assumptions. For example, nodes have to synchronize wake-up schedules among other nodes in the network [20-22].

In order to address the limitations of existing work, we propose a delay-constrained duty-cycle scheduling (DDS) algorithm with the objective of achieving low energy consumption while satisfying the delay bound requirements in duty-cycled WSNs. DDS does not require time or wake-up schedule synchronization. Moreover, no specific node deployment strategy is required, i.e., sensor nodes can be placed inside buildings or outdoors, following a deterministic or nondeterministic deployment layout depending on user application demands.

In the considered WSNs, data packets are required to reach the sink within a given time; i.e., the E2E delay of packets is bounded by an application-specific demand. For example, an application may require $95 \%$ of transmitted packets to arrive at the sink within $30 \mathrm{~s}$ [21,23]. More formally, in this work, a deadline success ratio (DSR) is defined as the ratio between the number of packets delivered to the sink within the given delay bound and the total number of transmitted packets (e.g., the application may require a $95 \%$ DSR with a delay bound of $30 \mathrm{~s}$ ).

Under DDS, after sensor nodes are deployed, they are assigned a group number based on the hop distance to the sink. To deliver data packets to the sink, sensor nodes in a group forward their packets to the first awake node from among potential forwarders that belong to a group closer to the sink. The sink collects the network parameters (e.g., the number of nodes in each group, the number of potential forwarders, and the data generation rate). Using the collected information, the one-hop delay distribution of each group is obtained as a function of the duty cycle interval, the delay bound, and the required DSR. Since our work considers custom node deployment, one-hop delay at nodes in different groups may have different distributions. So, we cannot apply the classical central limit theorem [24] to the network scenario under consideration. Note that the Lyapunov central limit theorem [24] is used to estimate the distribution of a sum of random variables which can be non-identically distributed. Therefore, the DDS scheme uses the Lyapunov theorem to determine the E2E delay distribution. Finally, the maximal duty cycle interval that satisfies the given delay bound requirement is determined by using the obtained E2E delay distribution.

We also note that the proposed algorithm can overcome the limitations of our previous work [25], which assumed that nodes are randomly deployed following a uniform distribution in a circular area. By estimating the delay distribution based on the collected network topology information, the algorithm in this work is suitable for general cases where node deployment scenarios depend on various user demands. Moreover, existing studies [25,26] had to assume that one-hop delays of different node groups are identically distributed in order to use the classical central limit theorem. In contrast, this work allows each node group to have a different delay distribution by proving the 
Lyapunov condition and using the Lyapunov central limit theorem [24], which results in a higher performance in practical WSN scenarios.

In order to validate the performance of DDS, we compare DDS with four algorithms: the optimal anycast packet-forwarding (OAPF) [16], the optimal geographical progress (OGP) [19], and two extended deadline-aware scheduling and forwarding (DASF) [25] algorithms. The proposed algorithm was evaluated using performance metrics, including the achieved DSR, the total energy consumption by nodes in the network, and the average E2E delay. Simulation results show that DDS outperforms other existing algorithms in terms of the delay requirements (i.e., the given delay bound and DSR) and energy consumption. Although the average E2E delay under DDS is longer than that of OAPF and OGP, DDS satisfies the delay requirements (i.e., the required DSR) in various test scenarios (e.g., varied delay bounds, required DSR, and event rates), while OAPF and extended DASF could not satisfy the delay requirement in some cases. Moreover, DDS achieves better energy efficiency than OGP.

In summary, the main contributions of our work are listed as follows:

- This work proposes the delay-constrained duty-cycle scheduling (DDS) algorithm to achieve low energy consumption while satisfying the delay requirement in duty-cycled WSNs. Unlike several existing works [7-12,20-22] which needed strict time synchronization, DDS does not require time synchronization nor wake-up synchronization. Moreover, in contrast to some studies which assumed a specific node deployment $[18,19,25]$ (i.e., uniformly random distribution), the proposed duty-cycle scheduling algorithm can be applied to WSNs with various node deployment scenarios.

- We define the problem of maximizing the duty cycle interval subject to the delay requirement in duty-cycled WSNs. The sink first collects the network topology information, on the basis of which the one-hop delay distribution is computed. Then, the E2E delay distribution is estimated as a function of the duty cycle interval using the Lyapunov central limit theorem, which allows nodes in different groups to have different delay distributions. Finally, the maximal duty cycle interval is determined using the estimated E2E delay distribution to minimize consumed energy while satisfying the delay requirement. To the best of our knowledge, this work is the first attempt to estimate the E2E delay distribution using the collected network topology information and the Lyapunov central limit theorem so that it allows different one-hop delay distributions for groups.

- The simulations were conducted to evaluate the proposed algorithm and compare with other related works. The evaluation results show that the proposed DDS scheme leads to better performance than existing algorithms with regard to the achieved DSR and energy consumption in a variety of network scenarios, including different delay bounds, required DSR, and event rates.

The rest of this paper is organized as follows. Section 2 introduces related work. Section 3 presents the network model and defines a maximization problem that aims to find the maximal duty cycle interval while satisfying the given delay bound requirement. Then, we describe the delay-constrained duty-cycle scheduling algorithm in detail in Section 4. Evaluation of the performance and a comparison with existing algorithms are shown in Section 5. Finally, this paper is concluded in Section 6.

\section{Related Work}

Duty cycling is an effective solution for reducing energy consumption in WSNs [11,27-33]. However, since a long E2E delay can be a critical problem in duty-cycled WSNs, there have been a lot of studies that considered the E2E delay.

Several studies [7-19] focused on minimizing the expected E2E delay. For example, Nazir et al. [14] proposed a sleep/wake scheduling scheme to minimize the E2E delay by increasing the active periods of the nodes according to the traffic load. Nodes are assigned different sleep/wake schedules considering three cases. The first is based on the node's distance from the sink, the second is the topological importance of the node (e.g., a node that is the forwarder for many nodes has a longer active duration), and the third is the occurrence of events (e.g., when an event occurs near the nodes, their active period increases). 
Kim et al. [16] proposed a sleep/wake scheduling policy that aims at minimizing the E2E delay and maximizing the lifetime of event-driven WSNs. In their study, nodes maintain a list of forwarding candidates, each of which is assigned a priority. When a node wants to transmit a packet, it checks the channel for a certain period of time to find forwarding nodes that wake up in that period. If there is only one forwarder awake, the transmitter transmits the packet to this node. In the case of multiple forwarders, the transmitter selects the forwarder that has the highest priority. The expected E2E delay at each node is calculated based on the awake probability, the forwarding candidates, and the priority of the forwarders. Then, OAPF is proposed to find an optimal forwarding policy that minimizes the expected E2E delay.

Wang et al. [19] proposed a geographic routing algorithm, called optimal geographical progress (OGP), in order to reduce the expected packet latency in duty-cycled WSNs. Each node maintains a set of possible forwarders that make geographical progress of at least a threshold and forwards the packets to the first wake-up node among candidate forwarders. Wang et al. showed that the threshold value has a great impact on the packet E2E delay, and a low latency can be obtained by using an appropriate threshold value.

Since those studies only considered the expected E2E delay, they could not guarantee or control the portion of the packets that arrive at the sink within the delay bound. Note that even when the expected E2E delay is shorter than the delay bound, a large portion of the packets might reach the sink after the delay bound due to the variance in E2E delay.

In contrast, our work estimates a probabilistic distribution of the E2E delay as a function of the duty cycle interval, the delay bound, and the required DSR. This enables the WSN to satisfy the delay bound constraint while lowering energy consumption by controlling the duty cycle interval.

It is worthwhile to note one study that considered E2E delay distribution [26]. However, its objective was only to estimate the E2E delay distribution using a given fixed routing path, rather than to control network parameters to improve network performance (e.g., satisfying the delay requirement or reducing energy consumption). In addition, it required random variables for one-hop delays to be identically distributed, which may not be practical in certain network scenarios.

A few studies [20-22,25] considered the delay bound requirement in WSNs. For example, Yang and Augé-Blum [20] proposed a wake-up scheduling-based forwarding scheme to satisfy the delay bound while the network achieves a high packet delivery ratio. This scheme schedules the active and dormant states of each node based on the hop count and the expected delivery ratio to the sink. This study is different from ours in that it attempted to improve the packet delivery ratio rather than reducing energy consumption. In addition, this scheme requires time synchronization between neighboring nodes.

Fan et al. proposed the delay-bounded transmission power control (DTPC) algorithm, which aims at minimizing the energy consumption of sensors while meeting the delay constraint [21]. With a stronger transmission power, the E2E delay can be reduced, but energy consumption may increase. Therefore, under DTPC, nodes select an optimal power level for the considered objective. However, they require network-wide time synchronization and wake-up schedule synchronization between neighboring nodes.

Dao et al. [25] took into consideration both the delay bound requirement and energy consumption. In this scheme, the E2E delay distribution is estimated using the duty cycle interval and the number of potential forwarders of nodes. It was shown that the delay bound requirement can be satisfied with low energy consumption by adjusting the duty cycle interval. However, this work required specific node deployment, i.e., nodes should be randomly deployed following a uniform distribution in a circular area, where the sink is placed at the center. In addition, it was also assumed that one-hop delays are identically distributed. In contrast, our work does not require a specific node deployment strategy or identically distributed one-hop delays, which makes it much more practical in various sensing scenarios. 
Table 1 summarizes the differences between our proposed work and existing studies which aimed at improving packet latency. As can be seen in Table 1, most existing studies focused on simply reducing the E2E delay, rather than considering the delay requirement [7-19]. That means that the studies in [7-19] could not guarantee the latency bound for delay-constrained applications.

In addition, there are some studies which have attempted to address the delay constraint problem in duty-cycled WSNs [20-22,25]. However, some algorithms [20-22] required strict time synchronization between nodes which could incur considerably high communication overhead for exchanging synchronization information in the network. Unlike the studies in [20-22], our proposed DDS algorithm does not require nodes to synchronize their wake-up schedules, leading to low packet overhead.

Moreover, even though time synchronization between sensor nodes is not needed, the applicability of the algorithm in [25] is restricted by the assumption that nodes are randomly deployed with a uniform distribution. In many practical scenarios where sensor nodes are placed based on specific application demands, this assumption may not be satisfied. Therefore, we aim at designing a duty-cycle scheduling algorithm which can be applied to WSNs with a custom node deployment.

In summary, in order to address the limitations of the existing studies, the delay-constrained duty-cycle scheduling (DDS) algorithm is proposed. Our work focuses on developing a duty-cycle scheduling algorithm which reduces energy consumption in the network while meeting the delay requirement in duty-cycled WSNs. Moreover, there is no time synchronization procedure, and the DDS scheme can be used for various node deployment scenarios.

Table 1. Comparison table between our proposed duty-cycle scheduling (DDS) and existing studies.

\begin{tabular}{cccc}
\hline Studies & Objective & Time Synchronization & Node Deployment Requirement \\
\hline$[7-12]$ & to minimize expected E2E delay & yes & no \\
{$[13-17]$} & to minimize expected E2E delay & no & no \\
{$[18,19]$} & to minimize expected E2E delay & no & uniformly random distribution \\
{$[20-22]$} & to satisfy the delay constraint & yes & no \\
{$[25]$} & to satisfy the delay constraint & no & uniformly random distribution \\
DDS & to satisfy the delay constraint & no & no \\
\hline
\end{tabular}

\section{Network Model and Problem Definition}

In this section, the network model and problem definition are described.

\subsection{Network Model}

This work considers a network of $(N-1)$ static sensor nodes and a sink node. Sensor nodes have the same transmission range, within which they can communicate with other nodes. The node deployment strategy can be varied based on specific user demands, i.e., sensors can be placed inside buildings or outdoors, following deterministic or nondeterministic node deployment layouts.

In order to reduce power consumption, the duty cycling mechanism is used. Nodes can turn off the radio and be dormant during the duty cycle interval except for the sink, which is always active. Nodes only transmit and receive data packets when they are in the active state. In addition, when a node in the sleep state detects an event, the node's radio is turned on to transmit packets. Let $T$ denote the duty cycle interval, and let $\tau$ denote the active period of nodes in a duty cycle interval. For each duty cycle interval, sensor nodes independently select a random time to wake up.

\subsection{Problem Definition}

Satisfying delay requirements is one of the most critical concerns in delay-constrained applications in WSNs. For such applications, data packets must reach the sink within a given time. Otherwise, the information in the packets might be useless. Therefore, E2E delay, which is the latency of packets from the source node to the sink, should be bounded according to specific application demands. 
This work considers both the delay bound and the required DSR, e.g., an application requires $95 \%$ of the transmitted packets (the required DSR) to arrive at the sink within $30 \mathrm{~s}$ (the given delay bound) [21,23].

Let $\theta$ and $\gamma$ denote a given delay bound and a required deadline success ratio of an application, respectively. Random variable $\mathrm{d}$ is defined as the packets' E2E delay, which is the sum of one-hop delays. In duty-cycled WSNs, as the duty cycle interval increases, energy consumption can be reduced. However, a large duty cycle interval leads to an increase in E2E delay. Therefore, the objective of the problem is to find the maximal duty cycle interval, $T$, subject to the constraint that the probability of $d$ being smaller than $\theta$ should be greater than $\gamma$. The detailed algorithm for finding the maximal value of $T$ is presented in Section 4.

\section{A Delay-Constrained Duty-Cycle Scheduling Algorithm}

In this section, a delay-constrained duty-cycle scheduling (DDS) algorithm is described. First, we present a group-based forwarding algorithm that consists of two phases. In the first phase, the sink collects network topology information. The second phase implements a scheduling algorithm for data transmission. For duty-cycle scheduling, using the information gathered during the first phase, the one-hop delay distribution of each group is formulated as a function of the duty cycle interval, the delay bound, and the required DSR. Then, the distribution of E2E delay, which is the sum of the one-hop delays of the groups, is estimated. Finally, the maximal duty cycle interval that satisfies the given delay bound requirement is determined by using the obtained E2E delay distribution.

\subsection{Group-Based Forwarding Algorithm}

The proposed scheme consists of two phases: the group determination phase and the data transmission phase. In the first phase, the group index of each node is assigned. Then, the sink collects the information necessary for duty cycle interval determination, such as the number of forwarding candidates and the number of nodes in each group. Nodes forward packets to the sink in the data transmission phase.

\subsubsection{Group Determination Phase}

This phase includes two subphases. The first one assigns the group index to the nodes, and the second one aggregates the network information at the sink.

First, the group index of the nodes is determined as follows. The group index of the sink is set to zero while sensor nodes initialize the infinite values for the group indices. Each node broadcasts a hello message (which contains the node's group index) periodically or when the group index of the node changes. When node A (except for the sink) receives a hello message from node B, the group index of node $\mathrm{A}$ is calculated as follows:

$$
G I_{A}= \begin{cases}G I_{B}+1, & \text { if } G I_{A}>G I_{B}+1, \\ G I_{A}, & \text { otherwise, }\end{cases}
$$

where $G I_{A}$ and $G I_{B}$ are the group indices of node $\mathrm{A}$ and node $\mathrm{B}$, respectively.

After updating their group index, nodes immediately broadcast the changed information to neighboring nodes. At the end of this subphase, nodes update the group index and maintain the forwarder's information, which will be sent to the sink in the second subphase.

Let $j$ denote the group index of nodes, i.e., nodes in group $j$ have a group index that equals $j$. Let $N_{j}$ and $g$ denote the number of nodes in group $j$ and the largest group index for nodes in the network, respectively. Thus, $j=0,1,2, \ldots, g$. Note that only the sink node is in group 0 . Let $n_{j}^{i}$ denote the $i$ th node in group $j$, where $i=1,2, \ldots, N_{j}$. We define the potential forwarding candidates (PFCs) of node $n_{j}^{i}$ as nodes that are in group $(j-1)$ within the transmission range of node $n_{j}^{i}$. 
Figure 1 shows a WSN that consists of a sink node and nine sensor nodes. By the first subphase, nodes know their group index, neighboring nodes, and potential forwarders. For example, node $n_{2}^{1}$ in group 2 has four neighboring nodes (i.e., $n_{3}^{1}, n_{2}^{2}, n_{1}^{2}$, and $n_{1}^{1}$ ) and two PFCs (i.e., $n_{1}^{1}$ and $n_{1}^{2}$ ).
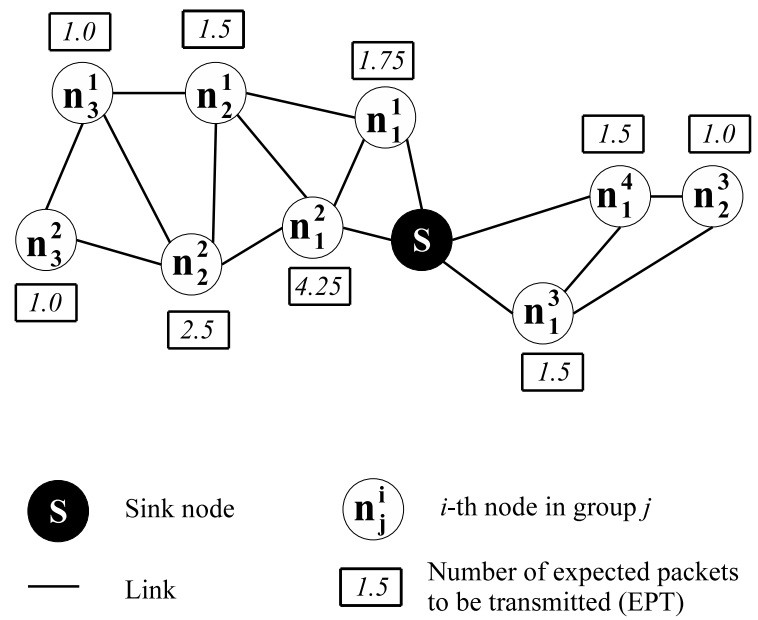

Figure 1. Example of group determination phase and expected packets to be transmitted.

In the second subphase, the sink collects the information necessary for estimating the duty cycle interval. Instead of using a flooding protocol that causes redundant packets, a period-based scheme is used. First, this subphase duration is divided into $q$ equal periods, which should be greater than or equal to the maximum group index (i.e., $q \geq g$ ). We assume that the sink knows and broadcasts the $q$ value throughout the network. In the first period, each node with a group index equal to $q$ (i.e., $j=q$ ) forwards an information packet that contains the necessary information (i.e., the group index and the list of PFCs) to a next-hop node. The next-hop node is randomly selected from among the PFCs of the transmitter. Then, nodes in group $(q-1)$ that received information packets append their information to the received information packets. Thus, nodes in group $(q-1)$ have information packets that contain information from nodes in group $q$ and $(q-1)$. With the same procedure, at the beginning of the second period, nodes in group $(q-1)$ forward their information packets to the next-hop nodes that are in group $(q-2)$. The second subphase finishes when the nodes in group 1 forward their information packets to the sink.

For instance, assume that $q$ is set to 3 in the network deployment scenario shown in Figure 1. At the beginning of the first period, nodes in group 3 forward their information packets to nodes in group 2. Then, each node in group 2 appends its information to the received information packets from nodes in group 3 . These combined packets are relayed to nodes in group 1 at the beginning of the second period. Finally, the sink receives network information, including the number of groups in the network (i.e., $g=3$ ), the number of nodes in the groups, and the PFCs of the nodes. The information from group 2 is shown in Table 2.

Table 2. The information from nodes in group 2.

\begin{tabular}{cccc}
\hline Node & Group Index & Number of PFCs & List of PFCs \\
\hline$n_{2}^{1}$ & 2 & 2 & $n_{1}^{1}, n_{1}^{2}$ \\
$n_{2}^{2}$ & 2 & 1 & $n_{1}^{2}$ \\
$n_{2}^{3}$ & 2 & 2 & $n_{1}^{3}, n_{1}^{4}$ \\
\hline
\end{tabular}




\subsubsection{Data Transmission Phase}

In this phase, data packets are transmitted to the sink. When an event is detected near a node, this node packs information into a data packet and transmits the packet to the first awake node among its PFCs.

Generally, sensor nodes wake up according to a Poisson process or when an event is detected. With Poisson wake-up scheduling, we divide the half-line $[0, \infty)$ of the working timeline of nodes into subintervals of length $T$. Each node independently wakes up once at a random time during a duty cycle interval and broadcasts a beacon message to announce that it is available to relay packets. Thus, the wake-up rate for nodes is $\frac{1}{T}$. The time that nodes wake up in a duty cycle interval follows a uniform distribution. The duty cycle interval $T$ is divided into $\epsilon$ equal time slots, where $\epsilon$ is the number of subintervals, $\frac{T}{\epsilon}$. The wake-up time for each node in the $l^{\text {th }}$ duty cycle interval (i.e., $[(l-1) \times T, l \times T), l=1,2,3, \ldots)$ is calculated as follows:

$$
t_{l^{\text {th }}}=(l-1) T+\frac{\operatorname{rand}(0, \epsilon-1)}{\epsilon} T
$$

where $\operatorname{rand}(0, \epsilon-1)$ returns a uniformly distributed random integer number in the range $[0, \epsilon-1]$.

When detecting an event, a node becomes a transmitter and waits for beacon messages from its PFCs. Then, this transmitter relays data packets to the first awake node among its potential forwarders and waits for an acknowledge (ACK) message from the receiver. When the ACK is successfully received, the transmission ends. Otherwise, the transmitter backs off for a random period, and then retransmits the packet to the receiver. Packets are discarded when the number of retransmissions exceeds a given upper bound.

Figure 2 shows an example of transmission between nodes A and B. Node B sends a beacon message after waking up and stays in the idle state, in which the node stays awake but does not transmit or receive packets. After receiving the beacon, node A checks whether or not node B is a PFC of node A. If so, node A forwards data packets to node B, which is the first awake node among node A's PFCs, and then waits for an ACK from node B.

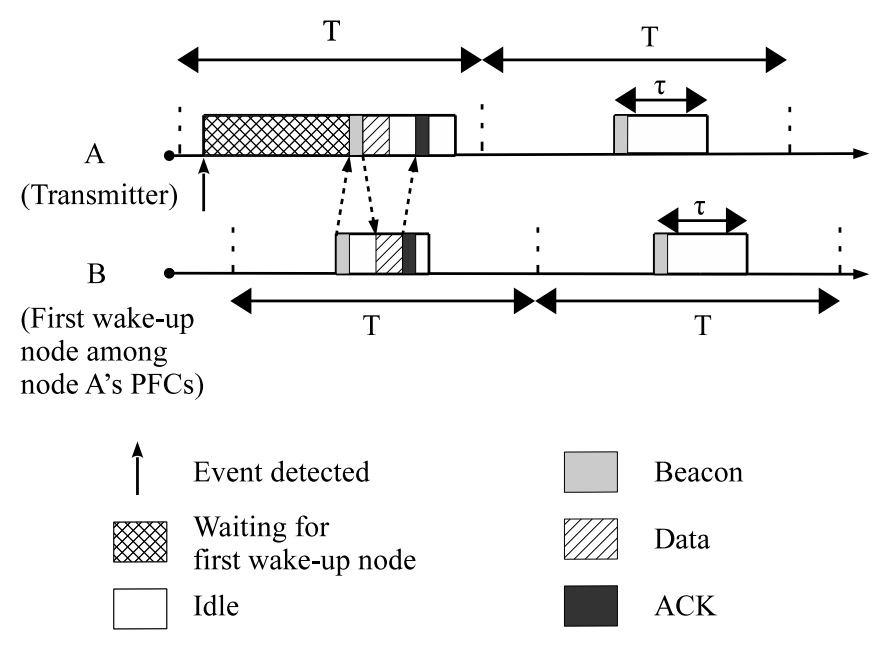

Figure 2. Example of data transmission phase.

\subsection{Estimation of One-Hop Delay Distribution}

Since the E2E delay is the sum of the one-hop delays, estimating the one-hop delay distribution of each group is necessary to obtain the E2E delay distribution. The one-hop delay of packets depends on the active/dormant schedules of the transmitters' PFCs. With a larger number of PFCs, the one-hop delay can be shorter. In addition, nodes transmit not only their own packets but also packets received 
from other nodes. Thus, nodes may transmit a different number of packets from the others. Note that the one-hop delay experienced by a group also depends on the number of packets to be transmitted. For instance, assume that nodes $\mathrm{A}$ and $\mathrm{B}$ in group 3 have to transmit 10 packets to nodes in group 2 , and the one-hop delays of nodes $\mathrm{A}$ and $\mathrm{B}$ are $1 \mathrm{~s}$ and $10 \mathrm{~s}$, respectively. If the number of packets to be transmitted by nodes $A$ and $B$ are the same, the sum of one-hop delays for the 10 packets is $5 \times 1+5 \times 10=55 \mathrm{~s}$, and the average is $5.5 \mathrm{~s}$. On the other hand, if nodes A and B transmit nine packets and one packet, respectively, the sum of one-hop delays for the 10 packets is $9 \times 1+1 \times 10=19 \mathrm{~s}$, and the average will be $1.9 \mathrm{~s}$. Therefore, we consider both the number of PFCs and the number of packets to be transmitted in order to estimate the one-hop delay distributions of the groups.

To facilitate the discussion, suppose that every node generates only one data packet destined for the sink. Let expected packet(s) to be transmitted (EPT) denote the number of packets that a node has to forward to the sink. Let $u_{j}^{i}$ and $v_{j}^{i}$ denote the number of PFCs and the EPT of node $n_{j}^{i}$, respectively. As a result, the $i$ th node in group $j$ has to transmit $v_{j}^{i}$ packets to the earliest awake node from among $u_{j}^{i}$ potential forwarders. The EPT for node $n_{j}^{i}$ depends on the number of node $n_{j}^{i}$ s PFCs, as follows:

$$
v_{j}^{i}=1+\sum_{k=1}^{N_{j+1}} \alpha_{j}^{k} \frac{v_{j+1}^{k}}{u_{j+1}^{k}}
$$

where 1 is the number of packets generated at node $n_{j}^{i}$. The term $\sum_{k=1}^{N_{j+1}} \alpha_{j}^{k} \frac{v_{j+1}^{k}}{u_{j+1}^{k}}$ represents the number of packets received from other nodes. $\alpha_{j}^{k}$ is a coefficient with respect to node $n_{j}^{k}$. If node $n_{j}^{i}$ is a PFC of node $n_{j+1}^{k}, \alpha_{j}^{k}=1$; otherwise, $\alpha_{j}^{k}=0$.

Figure 1 also shows the EPT for each node in the network. Nodes $n_{3}^{1}$ and $n_{3}^{2}$ in group 3 generate one packet and do not receive any packets from the others. Thus, node $n_{3}^{1}$ and $n_{3}^{2}$ have the same EPT, i.e., $v_{3}^{1}=v_{3}^{2}=1$. . Since node $n_{2}^{1}$ only forwards packets from node $n_{3}^{1}$ and $u_{3}^{1}=2$, the EPT for node $n_{2}^{1}$ is $v_{2}^{1}=1.0+\frac{v_{3}^{1}}{u_{3}^{1}}=1.0+\frac{1.0}{2}=1.5$ packets.

Let us define $P\left(n_{j}^{i}\right)$ as the probability that an arbitrary packet in group $j$ is forwarded by node $n_{j}^{i}$. With a larger EPT for node $n_{j}^{i}$, the probability that this node transmits a packet is higher. Therefore, $P\left(n_{j}^{i}\right)$ depends on the EPT of the nodes, and can be expressed as follows:

$$
P\left(n_{j}^{i}\right)=\frac{v_{j}^{i}}{\sum_{k=1}^{N_{j}} v_{j}^{k}}
$$

As shown in Figure 1, nodes $n_{2}^{1}, n_{2}^{2}$, and $n_{2}^{3}$ in group 2 have 1.5 EPT, 2.5 EPT, and 1.0 EPT to forward, respectively. Thus, the total number of packets to be transmitted by the nodes in group 2 are $1.5+2.5+1.0=5.0$ packets. According to Equation (4), the probabilities that nodes $n_{2}^{1}, n_{2}^{2}$, and $n_{2}^{3}$ transmit packets is $\frac{1.5}{5.0}=0.3, \frac{2.5}{5.0}=0.5, \frac{1.0}{5.0}=0.2$, respectively.

Then, the one-hop delay distribution of each group is obtained based on the number of PFCs and the EPT. Let $h_{j}$ denote the random variable for the one-hop delay of group $j$, and let $h_{j}$ be a given value for random variable $\mathrm{h}_{j}$. Let $P\left(\mathrm{~h}_{j} \leq h_{j} \mid n_{j}^{i}\right)$ denote the probability that the one-hop delay of an arbitrary packet to be transmitted by node $n_{j}^{i}$ is not larger than $h_{j}$. A random variable, $\mathrm{w}_{j}^{k}$, is defined as the wake-up time for node $n_{j}^{k}$ from among $u_{j}^{i}$ PFCs of node $n_{j}^{i}$ during a duty cycle interval where $k=1,2, \ldots, u_{j}^{i}$. Let $w_{j}^{k}$ denote a given value for random variable $w_{j}^{k}$. Since nodes wake up once, and they independently choose a random wake-up time in each duty cycle interval, the probability that node $n_{j}^{k}$ wakes up before $w_{j}^{k}$ is $P\left(\mathrm{w}_{j}^{k} \leq w_{j}^{k}\right)=\frac{w_{j}^{k}}{T}$ where $0 \leq w_{j}^{k} \leq T$. Furthermore, node $n_{j}^{i}$ selects the first wake-up node from among $u_{j}^{i}$ nodes. Therefore, the one-hop delay of a packet transmitted by node $n_{j}^{i}$ is the earliest wake-up time of $u_{j}^{i}$ nodes. 
The probability $P\left(\mathrm{~h}_{j}>h_{j} \mid n_{j}^{i}\right)$, in which the one-hop delay of an arbitrary packet to be transmitted by node $n_{j}^{i}$ is greater than $h_{j}$, equals the probability that $u_{j}^{i}$ forwarding candidates of node $n_{j}^{i}$ wake up after $h_{j}$, and it can be expressed as follows:

$$
\begin{aligned}
P\left(\mathrm{~h}_{j}>h_{j} \mid n_{j}^{i}\right) & =\prod_{k=1}^{u_{j}^{i}} P\left(\mathrm{w}_{j}^{k}>h_{j}\right)=\prod_{k=1}^{u_{j}^{i}}\left[1-P\left(\mathrm{w}_{j}^{k} \leq h_{j}\right)\right]=\prod_{k=1}^{u_{j}^{i}}\left(1-\frac{h_{j}}{T}\right) \\
& =\left(1-\frac{h_{j}}{T}\right)^{u_{j}^{i}}
\end{aligned}
$$

Therefore, the probability that the one-hop delay of an arbitrary packet to be forwarded by node $n_{j}^{i}$ will not be greater than $h_{j}$ can be calculated as follows:

$$
\begin{aligned}
F\left(h_{j} \mid n_{j}^{i}\right) & =P\left(\mathrm{~h}_{j} \leq h_{j} \mid n_{j}^{i}\right)=1-P\left(\mathrm{~h}_{j}>h_{j} \mid n_{j}^{i}\right) \\
& =1-\left(1-\frac{h_{j}}{T}\right)^{u_{j}^{i}}
\end{aligned}
$$

From Equations (4) and (6), the marginal probability mass function of the one-hop delay, $F\left(h_{j}\right)$ of group $j$, can be calculated using the law of total probability as follows:

$$
F\left(h_{j}\right)=\sum_{k=1}^{N_{j}} F\left(h_{j} \mid n_{j}^{k}\right) P\left(n_{j}^{k}\right)=\sum_{k=1}^{N_{j}}\left[1-\left(1-\frac{h_{j}}{T}\right)^{u_{j}^{k}}\right] P\left(n_{j}^{k}\right)
$$

According to Equation (7), the probability density function $f\left(h_{j}\right)$ is expressed as follows:

$$
f\left(h_{j}\right)=\frac{d F\left(h_{j}\right)}{d h_{j}}=\sum_{k=1}^{N_{j}} \frac{u_{j}^{k}}{T}\left(1-\frac{h_{j}}{T}\right)^{u_{j}^{k}-1} P\left(n_{j}^{k}\right)
$$

Mean $\mu_{j}$ and variance $\sigma_{j}^{2}$ of one-hop delay $\mathrm{h}_{j}$ are calculated as follows:

$$
\begin{gathered}
\mu_{j}=E\left[\mathrm{~h}_{j}\right]=\int_{0}^{T} h_{j} f\left(h_{j}\right) d h_{j}=T \sum_{k=1}^{N_{j}} \frac{P\left(n_{j}^{k}\right)}{u_{j}^{k}+1} \\
=\psi_{j} T \\
\sigma_{j}^{2}=E\left[\left(\mathrm{~h}_{j}-\mu_{j}\right)^{2}\right]=E\left[\mathrm{~h}_{j}^{2}\right]-E\left[\mathrm{~h}_{j}\right]^{2}=\int_{0}^{T} h_{j}^{2} f\left(h_{j}\right) d h_{j}-\mu_{j}^{2} \\
=T^{2} \sum_{k=1}^{N_{j}}\left[\frac{2 P\left(n_{j}^{k}\right)\left(u_{j}^{k}+1\right)-\left(u_{j}^{k}+2\right)\left(P\left(n_{j}^{k}\right)\right)^{2}}{\left(u_{j}^{k}+1\right)^{2}\left(u_{j}^{k}+2\right)}\right] \\
=\omega_{j}^{2} T^{2}
\end{gathered}
$$

We obtain $\psi_{j}$ and $\omega_{j}$ using the information collected in the group determination phase, as follows:

$$
\begin{aligned}
\psi_{j} & =\sum_{k=1}^{N_{j}} \frac{P\left(n_{j}^{k}\right)}{u_{j}^{k}+1} \\
\omega_{j}^{2} & =\sum_{k=1}^{N_{j}}\left[\frac{2 P\left(n_{j}^{k}\right)\left(u_{j}^{k}+1\right)-\left(u_{j}^{k}+2\right)\left(P\left(n_{j}^{k}\right)\right)^{2}}{\left(u_{j}^{k}+1\right)^{2}\left(u_{j}^{k}+2\right)}\right]
\end{aligned}
$$

\subsection{Duty Cycle Interval Determination}

When estimating the E2E delay distribution, previous researchers $[25,26]$ used the classical central limit theorem [24] to approximate the E2E delay distribution. To this end, they assumed that the 
random variables for the one-hop delay of different groups are identically distributed. As a result, the node deployments for the groups have to be the same, which may not be practical in many WSN deployment cases.

In order to address this limitation, we estimate the E2E delay distribution using the Lyapunov central limit theorem [24], which does not require random variables for the one-hop delay of groups to be identically distributed. As a result, our work is more suitable for various node deployment scenarios, e.g., in buildings or outdoors, in deterministic or nondeterministic node deployment layouts.

In this section, we first use the Lyapunov central limit theorem to approximate the E2E delay distribution. Then, the duty cycle interval is inferred from the obtained E2E delay distribution.

Since the sink is always active, the one-hop delay of the first hop is zero. If packets that have the longest E2E delay satisfy the delay bound, all packets in the network can satisfy the delay bound. Therefore, we consider the packets' E2E delay, which is transmitted by nodes in group $g$ and expressed as follows:

$$
\mathrm{d}=\sum_{k=2}^{g} \mathrm{~h}_{k}
$$

where $h_{2}, h_{3}, \cdots, h_{g}$ are independently and non-identically distributed random variables. Note that the one-hop delay is less than the duty cycle interval $\left|\mathrm{h}_{k}\right| \leq T \forall k$.

Each one-hop delay $\mathrm{h}_{j}$ has the mean $\mu_{j}$ and variance $\sigma_{j}^{2}$, which are determined by Equations (9) and (10), respectively. Let $\mathrm{r}_{j}=\mathrm{h}_{j}-\mu_{j}$. The mean and variance of a random variable $\mathrm{r}_{j}$ are calculated as follows:

$$
\begin{aligned}
& E\left[\mathrm{r}_{j}\right]=E\left[h_{j}\right]-\mu_{j}=0 \\
\operatorname{Var}\left[\mathrm{r}_{j}\right]= & E\left[\left(\mathrm{r}_{j}-E\left[\mathrm{r}_{j}\right]\right)^{2}\right]=E\left[\mathrm{r}_{j}^{2}\right]-E\left[\mathrm{r}_{j}\right]^{2} \\
= & E\left[\mathrm{r}_{j}^{2}\right]=E\left[\left(\mathrm{~h}_{j}-\mu_{j}\right)^{2}\right] \\
= & \sigma_{j}^{2}
\end{aligned}
$$

Let $s_{g}$ be calculated as follows:

$$
s_{g}^{2}=\sum_{k=2}^{g} \operatorname{Var}\left[\mathrm{r}_{k}\right]^{2}=\sum_{k=2}^{g} E\left[\mathrm{r}_{k}^{2}\right]=\sum_{k=2}^{g} \sigma_{k}^{2}
$$

The Lyapunov central limit theorem states that if $\left\{r_{2}, r_{3}, \ldots r_{g}\right\}$ is a sequence of independent random variables that has a zero mean and a finite variance, and if the Lyapunov condition holds, the sum of $\frac{r_{k}}{s_{g}}$ converges to the standard normal distribution as $g$ approaches infinity:

$$
\sum_{k=2}^{g} \frac{\mathrm{r}_{k}}{s_{g}} \rightarrow \mathcal{N}(0,1)
$$

The Lyapunov condition for a sequence of $\left\{\mathrm{r}_{2}, \mathrm{r}_{3}, \ldots, \mathrm{r}_{g}\right\}$ is expressed as follows:

$$
\lim _{g \rightarrow \infty} \sum_{k=2}^{g} \frac{1}{s_{g}^{2+\delta}} E\left[\left|\mathbf{r}_{k}\right|^{2+\delta}\right]=0
$$

where $E\left[\left|\mathbf{r}_{k}\right|^{2+\delta}\right]$ is the $(2+\delta)^{\text {th }}$ moment of the random variable $\mathbf{r}_{k}$ for some $\delta>0$.

Therefore, we first prove that random variables $\left\{\mathrm{r}_{2}, \mathrm{r}_{3}, \ldots, \mathrm{r}_{g}\right\}$ satisfy the Lyapunov condition with the following lemma. 
Lemma 1. Let $\left\{r_{2}, r_{3}, \ldots, r_{g}\right\}$ be independent random variables for which $\left|r_{j}\right| \leq T$ with $j=2,3, \ldots, g$ and $\lim _{g \rightarrow \infty} \frac{T}{s_{g}}=0$. Then, $\left\{r_{2}, r_{3}, \ldots, r_{g}\right\}$ satisfy the Lyapunov condition.

Proof. Since $\mathrm{h}_{j}$ is the one-hop delay of group $\mathrm{j}$, we have $0 \leq \mathrm{h}_{j} \leq T$ and $0 \leq \mu_{j} \leq T$. In other words, $\mathrm{h}_{j}-\mu_{j} \leq T$ and $\mathrm{h}_{j}-\mu_{j} \geq-T$. Thus, $\mathrm{r}_{j}$ is bounded by $T$ with $2 \leq j \leq g$ :

$$
\left|\mathrm{r}_{j}\right|=\left|\mathrm{h}_{j}-\mu_{j}\right| \leq T
$$

For $\delta=1$, the left side of the Lyapunov condition in Equation (17) can be expressed as follows:

$$
\begin{gathered}
0 \leq \sum_{k=2}^{g} \frac{1}{s_{g}^{3}} E\left[\left|\mathrm{r}_{k}\right|^{3}\right] \leq \sum_{k=2}^{g} \frac{E\left[\left|\mathrm{r}_{k}\right|^{2} T\right]}{s_{g}^{3}} \\
\Rightarrow \lim _{g \rightarrow \infty} 0 \leq \lim _{g \rightarrow \infty} \sum_{k=2}^{g} \frac{1}{s_{g}^{3}} E\left[\left|\mathrm{r}_{k}\right|^{3}\right] \leq \lim _{g \rightarrow \infty} \sum_{k=2}^{g} \frac{E\left[\left|\mathrm{r}_{k}\right|^{2} T\right]}{s_{g}^{3}}
\end{gathered}
$$

By Equation (15), $s_{g} \rightarrow \infty$ as $g \rightarrow \infty$. Therefore, $\lim _{g \rightarrow \infty} \frac{T}{s_{g}}=0$. We have:

$$
\lim _{g \rightarrow \infty} \sum_{k=2}^{g} \frac{E\left[\left|\mathbf{r}_{k}\right|^{2} T\right]}{s_{g}^{3}}=\lim _{g \rightarrow \infty} T \frac{s_{g}^{2}}{s_{g}^{3}}=\lim _{g \rightarrow \infty} \frac{T}{s_{g}}=0
$$

According to Equations (20) and (21), the limit of the left side of the Lyapunov condition in Equation (17) becomes:

$$
0 \leq \lim _{g \rightarrow \infty} \frac{1}{s_{g}^{3}} \sum_{k=2}^{g} E\left[\left|\mathbf{r}_{k}\right|^{3}\right] \leq 0
$$

Therefore, the Lyapunov condition holds when $\delta=1$.

Since the statement in Lemma 1 is satisfied, Equation (16) holds. Let us define the random variable $\mathrm{z}$ that is a function of the required DSR $\gamma$ as follows:

$$
\begin{aligned}
\mathrm{z} & =\sum_{k=2}^{g} \frac{\mathrm{r}_{k}}{s_{g}}=\sum_{k=2}^{g} \frac{\left(\mathrm{h}_{k}-\mu_{k}\right)}{s_{g}} \\
& =\frac{\mathrm{d}-\sum_{k=2}^{g} \mu_{k}}{s_{g}}=\frac{\mathrm{d}-T \sum_{k=2}^{g} \psi_{k}}{T \sum_{k=2}^{g} \omega_{k}}
\end{aligned}
$$

Thus, $\mathrm{z} \rightarrow \mathcal{N}(0,1)$, in which $\mathrm{z}$ is derived from the standard normal table with a given required DSR $\gamma$, e.g., with $\gamma=95 \%, z=1.65$.

Replacing $\mathrm{d}$ by the delay bound $\theta$, in order to satisfy the delay bound requirement with the given probability, the duty cycle interval is expressed as follows:

$$
T \leq \frac{\theta}{\sum_{k=2}^{g} \psi_{k}+\mathrm{z} \sum_{k=2}^{g} \omega_{k}}
$$

The maximum value of the duty cycle interval, $T_{\max }$, is obtained as follows:

$$
T_{\max }=\frac{\theta}{\sum_{k=2}^{g} \psi_{k}+\mathrm{z} \sum_{k=2}^{g} \omega_{k}}
$$

where $\psi_{k}$ and $\omega_{k}$ are calculated from Equation (11). 


\section{Performance Evaluation}

This section presents the performance analysis of the proposed algorithm. First, the simulation configuration is described. Then, we compare DDS with OAPF [16], OGP [19], and two extended algorithms of DASF [25].

\subsection{Simulation Configuration}

This work considers a WSN with node deployment following the Motescope testbed [34] called the Soda network. As seen in Figure 3, 78 nodes were deployed in a $40 \times 50 \mathrm{~m}$ area, where the dark star and the circles represent the sink and the sensor nodes, respectively. With this node deployment, the maximum group index in the Soda network is 6 (i.e., $g=6$ ).

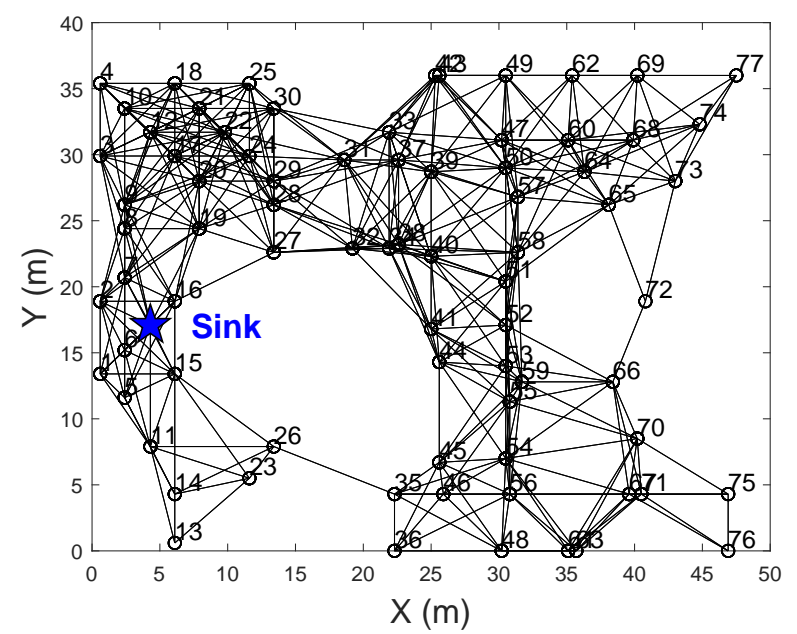

Figure 3. In the Soda network, 78 nodes were deployed following the Motescope testbed in 2007 in Soda Hall, University of California, Berkeley, California, USA.

The simulation setup is listed in Table 3. Sensor nodes are equipped with a Preon32 wireless module [35]. The transmission range and bandwidth of the sensors are $10 \mathrm{~m}$ and $2 \mathrm{Mbps}$, respectively. The power consumption of the Preon32 in the sleep, receiving, and transmitting modes is $60 \mathrm{nW}$, $36.9 \mathrm{~mW}$, and $72 \mathrm{~mW}$, respectively. The initial energy of each node is set to $500 \mathrm{~J}$, which is enough to ensure that no node consumes all energy before the simulation finishes. In the data transmission phase, an event randomly occurs at an arbitrary node. The number of events occurring in a given time follows the Poisson distribution with a mean of $\lambda$. For example, if the event rate is set to 0.5 packets $/ \mathrm{s}$, there is 0.5 event per $1 \mathrm{~s}$ on average, i.e., $\lambda=0.5$. Then, this node packs information into a 46 -byte data packet to transmit to the sink. During a duty cycle interval, nodes are awake for the active period $\tau=0.4 \mathrm{~s}$. We used the $n s-2$ network simulator [36] version 2.35 for the simulation, in which the final result is the average of 30 execution results. The main features for the transmission model setup in ns-2 are also shown in Table 3.

Network performance was analyzed under the evaluation parameters shown in Table 4. In order to evaluate the effects of each evaluation parameter on network performance, only the value of one selected parameter was varied, while the other parameters remained at default values. The default values for the delay bound, the required DSR, and the event rate were $30 \mathrm{~s}, 95 \%$, and 0.5 packet/s, respectively. 
Table 3. Simulation parameters.

\begin{tabular}{cc}
\hline Parameter & Value \\
\hline Sensing area & $40 \times 50 \mathrm{~m}$ \\
Transmission range & $10 \mathrm{~m}$ \\
Bandwidth & $2 \mathrm{Mbps}$ \\
Number of nodes in area & 78 \\
Active period & $0.4 \mathrm{~s}$ \\
Data packet size & $46 \mathrm{bytes}$ \\
Data transmission phase duration & $50 \mathrm{~min}$ \\
Initial energy of nodes & $500 \mathrm{~J}$ \\
Radio propagation model & TwoRayGround \\
Antenna model & OmniAntenna \\
Interface queue type & PriQueue \\
\hline
\end{tabular}

Table 4. Evaluation parameters.

\begin{tabular}{ccc}
\hline Parameter & Range of Value & Default Value \\
\hline Delay bound (seconds) & $\{10,20,30,40,50\}$ & 30 \\
Required DSR (\%) & $\{80,85,90,95,97\}$ & 95 \\
Event rate (packets/s) & $\{0.1,0.5,1.0,2.0\}$ & 0.5 \\
\hline
\end{tabular}

\subsection{Compared Algorithms}

In this subsection, we present algorithms with which the proposed DDS scheme is compared: the optimal anycast packet forwarding (OAPF) [16], the optimal geographical progress (OGP) [19], and two extended DASF [25] algorithms. The objective of OAPF and OGP is to minimize E2E delay with a given duty cycle interval while the DASF algorithm aims at satisfying the delay constraint in the duty-cycled WSNs.

\subsubsection{OAPF}

In OAPF, transmitters periodically broadcast a beacon signal and an ID message that carries sender information, and then they wait for an ACK from neighboring nodes. Let us define $t_{B}, t_{C}$, and $t_{A}$ as the period for sending the beacon, the ID, and the ACK, respectively. Transmitters repeat this beacon-ID-ACK procedure until they receive ACKs. After a node receives a beacon, it awakes to receive the ID message to check whether it is in the forwarding set of the transmitter. If the node (i.e., receiver) is on the list of forwarders, it responds with an ACK to the sender. If the transmitter receives multiple ACKs, it selects one node based on the priority of nodes. Then, it transmits data packets. Let $t_{D}$ denote the transmission time for data packets. After receiving the data packets, the receiver becomes a transmitter and follows the beacon-ID-ACK procedure to find the next-hop relay for the data packets.

In OAPF, when a node wakes up, it can be selected as a forwarder for several transmitters. Therefore, there are potential collisions when transmitters attempt to forward packets to this node. In addition, since OAPF aims to reduce E2E delay, the transmission times for data packets should not be large, and there is no packet recovery mechanism after packet collisions. We set time periods for beacon signaling, ID signaling, ACK wait time, and data transmission to $0.01 \mathrm{~s}, 0.01 \mathrm{~s}, 0.02 \mathrm{~s}$, and $0.03 \mathrm{~s}$, respectively.

The duty cycle interval calculated by DDS was applied to OAPF, since OAPF does not have a mechanism to determine the duty cycle interval. In addition, the wake-up rate of nodes in OAPF was set to the same as that of DDS, which is $\frac{1}{T}$. 


\subsubsection{OGP}

In order to reduce the expected packet latency in duty-cycled WSNs, Wang et al. [19] proposed a geographic routing algorithm, called optimal geographical progress (OGP). Each node maintains a set of possible forwarders that make geographical progress of more than a threshold and forwards the packets to the first wake-up node from among possible forwarding candidates. Wang et al. showed that the threshold value has a great impact on the packet E2E delay, and a low latency can be obtained by using an appropriate threshold value. Note that OGP does not have a mechanism to determine the duty cycle interval, so we used the duty cycle interval computed by the proposed DDS scheme for the OGP algorithm.

\subsubsection{Two Extended DASF Algorithms}

DASF cannot be directly applied to our problem, since nodes must be randomly deployed following a uniform distribution in a circular pattern under DASF. Therefore, we extended the idea of DASF to find the maximum duty cycle interval for the considered problem. In those algorithms, nodes randomly wake up once in a duty cycle interval, and transmitters select the first awake node among the PFCs for forwarding.

First, the one-hop delay distribution of each group is obtained, as discussed in Section 4.2. Then, one representative one-hop delay distribution is determined using the one-hop delay distributions of the groups, and it is assumed that all groups have the same distribution (i.e., the representative one-hop delay distribution) to make them independently and identically distributed. Now, the E2E delay is the sum of the representative distributions and is approximated using the classical central limit theorem.

We considered two variants to approximate the E2E delay distribution (specifically, to determine the representative one-hop distribution) as follows.

- Sum of representative one-hop delay using maximum mean (SROD-MM): For the representative distribution, we selected the one-hop delay distribution of a group that has the maximum mean value among the existing one-hop delay distributions. For example, with the Soda node deployment, the representative distribution chosen by SROD-MM is the one-hop delay distribution of group 4.

- Sum of representative one-hop delay estimated by weighted average (SROD-WA): Unlike SROD-MM, instead of selecting the representative distribution from existing distributions, this method estimates a new distribution that can represent all distributions of the groups. This new distribution is determined as the weighted sum of the one-hop delays using a mixture model [37], in which weights are calculated according to the groups' EPT. Let $v_{j}$ denote the EPT of the nodes in group $j$. Thus, $v_{j}=\sum_{k=1}^{N_{j}} v_{j}^{k}$. Let $M$ denote the representative distribution, expressed as follows:

$$
M=\sum_{k=2}^{g} \pi_{k} f\left(\mathrm{~h}_{k}\right)
$$

where $\pi_{k}=\frac{v_{k}}{\sum_{k=2}^{g} v_{k}}$ and $\sum_{k=2}^{g} \pi_{k}=1$.

The mean and variance of mixture distribution $M$ are calculated as follows:

$$
\begin{aligned}
& \mu_{M}=\sum_{k=2}^{g} \pi_{k} \mu_{k} \\
& \sigma_{M}^{2}=\sum_{k=2}^{g} \pi_{k}\left(\mu_{k}^{2}+\sigma_{k}^{2}\right)-\mu_{M}^{2}
\end{aligned}
$$




\subsection{Evaluation Results}

In this section, in order to evaluate the performance of DDS, the achieved DSR, total energy consumption in the network, and the average E2E delay were collected. We compared the proposed algorithm with OAPF, OGP, SROD-MM, and SROD-WA under the effects of the delay bound, the required DSR, and the event rate.

\subsubsection{Effects of the Delay Bound}

To investigate the effect of the delay bound on the network performance, the delay bound was varied from $10 \mathrm{~s}$ to $50 \mathrm{~s}$. With a longer delay bound, the duty cycle interval increases, since the duty cycle interval is proportional to the delay bound, according to Equation (25).

As seen in Figure 4a, DDS achieves the best performance in terms of the DSR. The results for the DSR indicate that the influence of the delay bound on the achieved DSR of DDS, SROD-MM, SROD-WA, and OGP is not enormous, in contrast to that of OAPF.

As the delay bound increases, the achieved DSR from DDS, SROD-MM, SROD-WA, and OGP has a similar trend, which is relatively flat with a small difference (i.e., from $1 \%$ to $2 \%$ ). According to Equation (25), when the delay bound changes, the DSR can remain unchanged by adjusting the duty cycle interval. Thus, DDS can consistently obtain a high DSR. In addition, OGP can achieve a relatively high DSR since a retransmission mechanism is implemented in this algorithm.

Meanwhile, the DSR under OAPF dramatically drops from 95.7\% to 70.1\%. This results from the fact that collisions reduce the packet delivery ratio, leading to a low DSR. With a longer duty cycle interval, when a node wakes up, it can be selected as a forwarder by more than one transmitter. Unfortunately, this node can only relay the packets of one node at that time, and the packets of other transmitters might be dropped because of collisions. In addition, under OAPF, transmitters forward data packets over a short period and do not retransmit when transmission fails. As a result, the packet delivery ratio decreases, which causes the low DSR.

Furthermore, DDS, SROD-MM, and OGP satisfy the required DSR, whereas OAPF does not with a delay bound greater than $10 \mathrm{~s}$. Although SROD-WA uses the same approach as SROD-MM, the DSR under SROD-WA is low and does not satisfy the required DSR, because many collisions occur with the high duty cycle interval obtained by SROD-WA.

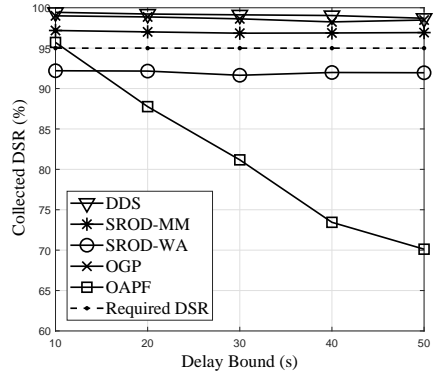

(a) Achieved DSR

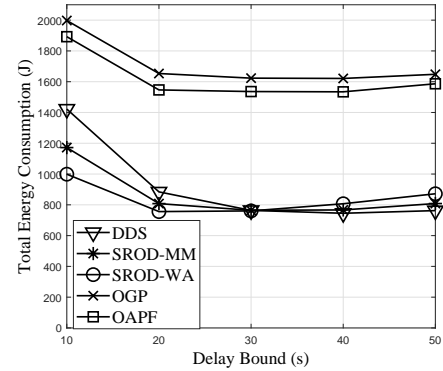

(b) Total energy consumption

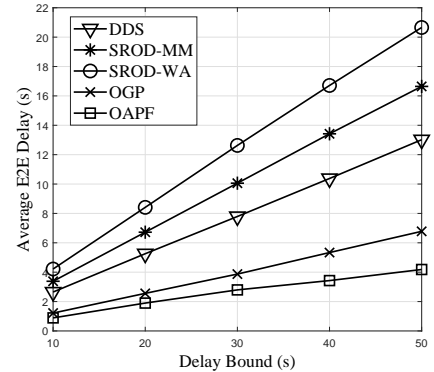

(c) Average E2E delay

Figure 4. Effects of delay bound on the achieved deadline success ration (DSR), total energy consumption in the network, and average end-to-end (E2E) delay, with required DSR $\gamma=95 \%$ and the event rate at 0.5 packet/s.

In terms of energy consumption, as shown in Figure 4b, DDS achieves lower energy consumption than SROD-MM, SROD-WA, OAPF, and OGP when the delay bound is greater than $30 \mathrm{~s}$. For instance, with a delay bound of $50 \mathrm{~s}$, SROD-MM, OAPF, and OGP spend 6.0\%, 108.1\%, and 116.2\% more energy than DDS, respectively.

Interestingly, the total energy consumption of the algorithms nonlinearly decreases as the delay bound increases. When the delay bound varies from $10 \mathrm{~s}$ to $20 \mathrm{~s}$, the increase in the duty cycle interval leads to a sharp decrease in energy consumption because the total number of active periods for the 
nodes decreases. On the other hand, when the delay bound increases from $20 \mathrm{~s}$ to $50 \mathrm{~s}$, with the larger duty cycle interval, nodes wake up less frequently. In this case, transmitters have to be active for a longer period to wait for their forwarding candidates, which leads to an increase in the total active periods of the transmitters. As a result, power consumption is relatively flat. Since the obtained duty cycle interval under DDS is the smallest, DDS achieves the best energy efficiency.

It should be noted that there is a big gap between the energy consumption of DDS and two counterpart algorithms: OGP and OAPF. That is because, under OAPF and OGP, nodes have to transmit data packets via more hops than under DDS. For example, the average hop count for packets under OAPF and OGP is around 4.2 and 4.5, respectively, whereas packets traverse to the sink in 3.3 hops under DDS. With a larger hop count, the number of intermediate nodes that have to forward data increases. Thus, energy consumption for the total active periods of the transmitters under OAPF and OGP is higher than that of DDS. Furthermore, transmitters under OAPF and OGP send more control packets than DDS. Therefore, OAPF and OGP consume more energy.

As shown in Figure 4c, the average E2E delay of the five schemes linearly increases and satisfies the given delay bound. As the duty cycle interval increases, the wake-up frequency of nodes is reduced. Transmitters have to wait longer for forwarding candidates to wake up. As a result, the one-hop delay, as well as the average E2E delay, increases. For instance, as the delay bound varies from $10 \mathrm{~s}$ to $50 \mathrm{~s}$, the average E2E delay with DDS linearly rises from $2.6 \mathrm{~s}$ to $13.0 \mathrm{~s}$, while the average E2E delay under OGP linearly increases from $1.2 \mathrm{~s}$ to $6.8 \mathrm{~s}$, respectively.

DDS achieves a lower average E2E delay than SROD-MM and SROD-WA because the obtained duty cycle interval under DDS is lower than that under SROD-MM and SROD-MA. However, the average E2E delay from DDS is longer than that from OAPF and OGP. The reason is attributed to the fact that OAPF and OGP use a short period to transmit data packets, i.e., OAPF and OGP focus on the average E2E delay rather than considering the DSR and energy consumption.

\subsubsection{Effects of the Deadline Success Ratio}

In order to evaluate the effects of the required DSR on network performance, we varied the DSR from $80 \%$ to $97 \%$ while the other parameters were kept to default values. With a higher required DSR $\gamma, \mathrm{z}$ in Equation (25) increases, since it is extracted from the standard normal table with the given $\gamma$. As a result, the duty cycle interval decreases.

With regard to the achieved DSR, as is shown in Figure 5a, DDS obtains the best performance when the required DSR is greater than $90 \%$. DDS, SROD-MM, and OGP satisfy the required DSR. On the other hand, SROD-WA does not satisfy a required DSR that is higher than $85 \%$. Even at the lowest DSR (i.e., 80\%), OAPF does not meet the requirement. For example, with a required DSR of 95\%, the achieved DSRs under DDS, SROD-WA, OAPF, and OGP are 99.1\%, $91.6 \%, 81.2 \%$, and $98.6 \%$, respectively.

It should be highlighted that the achieved DSR of the five schemes improves as the required DSR increases. However, OAPF obtains a low DSR due to the many collisions and the lack of a packet recovery mechanism. Although SROD-WA implements a packet recovery mechanism that is the same as that used by DDS, the achieved DSR under SROD-WA is still low because there are more collisions with a longer duty cycle interval. Meanwhile, since a retransmission mechanism is implemented in the OGP algorithm, the achieved DSR of OGP is relatively high.

As seen in Figure 5b, the energy consumption by DDS is approximated to two extended DASF algorithms and smaller than OAPF and OGP. For instance, when the required DSR is $95 \%$, the total energy consumption under DDS, SROD-MM, and SROD-WA is around $760 \mathrm{~J}$, while OAPF and OGP spend $101.2 \%$ and $109.7 \%$ more energy than DDS, respectively. Additionally, the total energy consumption by the schemes has a similar trend that is relatively flat as the required DSR increases (i.e., as the duty cycle interval decreases). This is because the decrement of the duty cycle interval has two opposing effects on energy consumption. First, with a shorter duty cycle interval, the energy consumed by the schemes increases because of the increase in the number of total awake periods 
for the nodes. Second, when the duty cycle interval decreases (i.e., the wake-up frequency for the nodes increases), the transmitters' waiting time for forwarding candidates is shorter. This leads to the decrease in energy consumption. As a result, the total energy consumption is relatively flat rather than notably increasing.

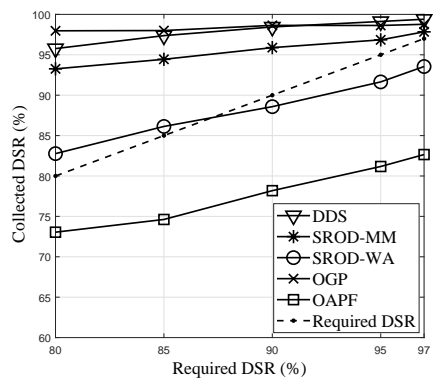

(a) Achieved DSR

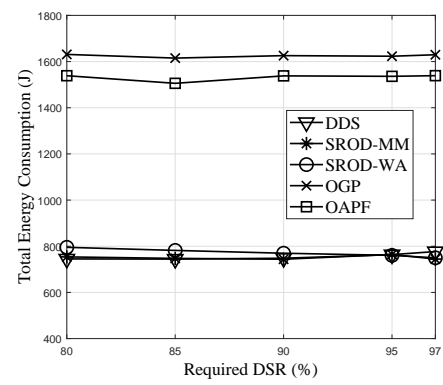

(b) Total energy consumption

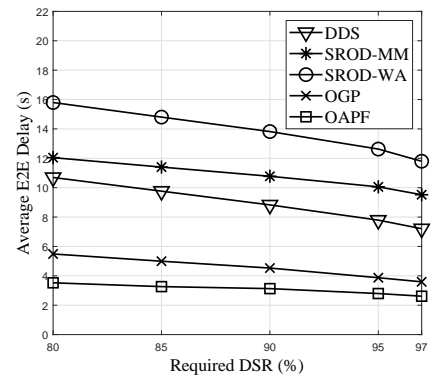

(c) Average E2E delay

Figure 5. Effects of the DSR on the achieved DSR, total energy consumption in the network, and average E2E delay with delay bound $\theta=30 \mathrm{~s}$ and an event rate of $0.5 \mathrm{packet} / \mathrm{s}$.

In terms of average E2E delay, DDS outperforms SROD-MM and SROD-WA, as shown in Figure 5c. The average E2E delay of the five schemes linearly decreases as the required DSR increases from $80 \%$ to $97 \%$. The duty cycle interval is reduced as the required DSR increases. With a lower duty cycle interval, nodes wake up more frequently, which leads to the decrease in E2E delay. For instance, as the required DSR varies from $80 \%$ to $97 \%$, the average E2E delay under DDS decreases from $10.7 \mathrm{~s}$ to $7.2 \mathrm{~s}$. With a required DSR of $90 \%$, the average E2E delay from DDS is $8.8 \mathrm{~s}$. This value is greater than 2.8 times the average E2E delay from OAPF and smaller than $18.1 \%$ of the average E2E delay from SROD-MM. The reason comes from the fact that OAPF and OGP aim to reduce the E2E delay by using a short period for transmitting data packets. On the other hand, the objective of DDS is to minimize energy consumption while satisfying the required DSR.

\subsubsection{Effects of the Event Rate}

The effects of the event rate on network performance are shown in Figure 6. The event rate was varied from 0.1 packets/s to 2.0 packets/s. Note that the duty cycle interval is constant in this case. The results indicate that the event rate significantly affects the achieved DSR and total energy consumption, whereas the average E2E delay is not affected by the event rate.

Figure 6a demonstrates the achieved DSR as the event rate increases from 0.1 packets/s to 2.0 packets/s. DDS still obtains the best performance and satisfies the required DSR. On the other hand, SROD-MM and OGP meet the requirement, while OAPF does not satisfy it with an event rate higher than 0.1 packets/s. In particular, SROD-WA cannot fulfill the required DSR, even at the lowest event rate (i.e., 0.1 packets/s).

In addition, the achieved DSR of the schemes tends to decrease as the event rate increases. For example, the achieved DSR under DDS goes from $99.4 \%$ to $98.6 \%$. Similarly, OAPF achieves DSRs that decrease from $96.1 \%$ to $60.9 \%$ while the achieved DSRs of OGP decrease from $99.5 \%$ to $96.2 \%$. This is because of a large number of collisions with the high network traffic.

In terms of power consumption, as observed in Figure 6b, DDS outperforms other schemes when the event rate is greater than 0.5 packets/s. For instance, with an event rate of 1.0 packets $/ \mathrm{s}$, the energy consumption of DDS, SROD-MM, OAPF, and OGP is $994.5 \mathrm{~J}, 1055.8 \mathrm{~J}, 1985.4 \mathrm{~J}$, and $1970.2 \mathrm{~J}$, respectively. In addition, the energy consumption of the schemes dramatically increases when the event rate rises because, with a larger number of generated packets, nodes have to be active longer to transmit packets. Recall that a longer active period leads to higher power consumption. In addition, OAPF and OGP 
consume more energy than DDS since the average hop count of packets under OAPF and OGP is larger than under DDS.

Figure $6 \mathrm{c}$ shows the effect of the event rate on the average E2E delay. The trend for the average E2E delay of the five schemes is relatively consistent. Since the average E2E delay is calculated for packets that are successfully transmitted to the sink, and the duty cycle interval is constant, the average E2E delay does not significantly change. DDS achieves a lower average E2E delay than SROD-MM and SROD-WA, because the duty cycle interval obtained under DDS is smaller than that with SROD-MM and SROD-WA. Since the transmission period in OAPF is short, OAPF achieves a lower average E2E delay than DDS. However, OAPF consumes the most energy and does not satisfy the required DSR when the event rate is higher than 0.1 packets/s.

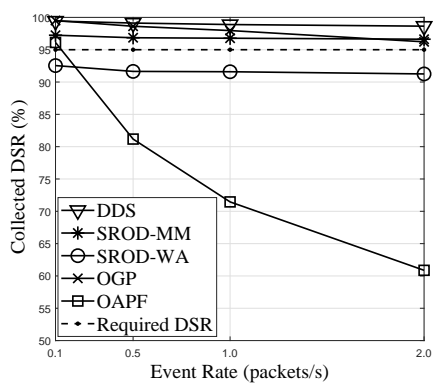

(a) Achieved DSR

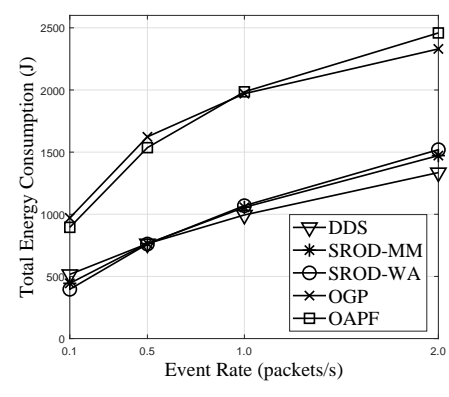

(b) Total energy consumption

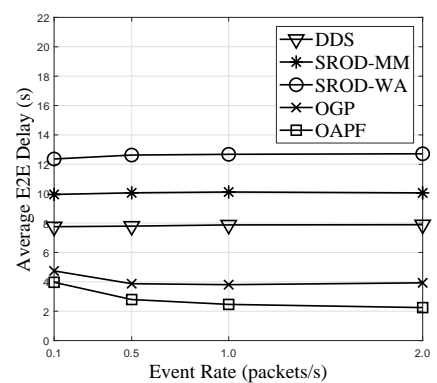

(c) Average E2E delay

Figure 6. Effects of event rate on the achieved DSR, total energy consumption in the network, and average E2E delay with delay bound $\theta=30 \mathrm{~s}$ and required DSR $\gamma=95 \%$.

\section{Conclusions}

In order to achieve low energy consumption while satisfying the delay bound requirements in duty-cycled WSNs, this paper proposes a novel scheduling algorithm. Using the network topology information collected by the sink, the one-hop delay distribution was derived as a function of the duty cycle interval, delay bound, and deadline success ratio. Then, the E2E delay distribution was estimated using the obtained one-hop delay and the Lyapunov central limit theorem. The duty cycle interval was determined using the obtained E2E delay distribution, with an objective of minimizing energy consumption while satisfying the delay bound requirement.

Unlike existing studies, our algorithm does not require the one-hop delays of groups to be identically distributed, which makes the algorithm applicable to various practical node-deployment scenarios. In addition, our work does not require time synchronization between nodes. Simulation results demonstrate that the proposed algorithm can satisfy the given delay bound requirements and outperforms existing algorithms in terms of the achieved deadline success ratio, total energy consumption, and the average E2E delay.

Author Contributions: Conceptualization, S.Y. and D.-S.V.; methodology, D.-S.V. and S.Y.; software, D.-S.V.; validation, D.-S.V., S.Y., and T.-N.D.; formal analysis, D.-S.V., T.-N.D., and S.Y.; investigation, D.-S.V. and S.Y.; resources, S.Y.; data curation, D.-S.V.; writing-original draft preparation, D.-S.V., T.-N.D., and S.Y.; writing-review and editing, D.-S.V., S.Y., and T.-N.D.; visualization, D.-S.V. and S.Y.; supervision, S.Y.; project administration, S.Y.; funding acquisition, S.Y.

Funding: This research was funded by the 2018 Research Fund of University of Ulsan.

Acknowledgments: The authors thank all editors and reviewers for their valuable comments, which have helped improve the manuscript.

Conflicts of Interest: The authors declare no conflict of interest. 


\section{References}

1. Ramson, S.R.J.; Moni, D.J. Applications of Wireless Sensor Networks-A Survey. In Proceedings of the 2017 International Conference on Innovations in Electrical, Electronics, Instrumentation and Media Technology (ICEEIMT), Coimbatore, India, 3-4 February 2017; pp. 325-329.

2. Minhas, U.I.; Naqvi, I.H.; Qaisar, S.; Ali, K.; Shahid, S.; Aslam, M.A. A WSN for Monitoring and Event Reporting in Underground Mine Environments. IEEE Syst. J. 2018, 12, 485-496. [CrossRef]

3. Taie, S.A.; Taha, S. A Novel Secured Traffic Monitoring System for VANET. In Proceedings of the 2017 IEEE International Conference on Pervasive Computing and Communications Workshops (PerCom Workshops), Kona, HI, USA, 13-17 March 2017; pp. 176-182.

4. Alfayez, F.; Hammoudeh, M.; Abuarqoub, A. A Survey on MAC Protocols for Duty-cycled Wireless Sensor Networks. Procedia Comput. Sci. 2015, 73, 482-489. [CrossRef]

5. Hao, J.; Zhang, B.; Mouftah, H.T. Routing Protocols for Duty cycled Wireless Sensor Networks: A Survey. IEEE Commun. Mag. 2012, 50, 116-123. [CrossRef]

6. Carrano, R.C.; Passos, D.; Magalhaes, L.C.S.; Albuquerque, C.V.N. Survey and Taxonomy of Duty Cycling Mechanisms in Wireless Sensor Networks. IEEE Commun. Surv. Tutor. 2014, 16, 181-194. [CrossRef]

7. Bacco, G.D.; Melodia, T.; Cuomo, F. A MAC Protocol for Delay-bounded Applications in Wireless Sensor Networks. In Proceedings of the MedHoc-Net, Bodrum, Turkey, 27-30 June 2004; pp. 208-220.

8. Sun, Y.; Du, S.; Gurewitz, O.; Johnson, D.B. DW-MAC: A Low Latency, Energy Efficient Demand-wakeup MAC Protocol for Wireless Sensor Networks. In Proceedings of the 9th ACM International Symposium on Mobile Ad Hoc Networking and Computing (MobiHoc '08), Hong Kong, China, 26-30 May 2008; ACM: New York, NY, USA, 2008; pp. 53-62.

9. Chatterjee, P.; Das, N. A Cross-layer Distributed TDMA Scheduling for Data Gathering with Minimum Latency in Wireless Sensor Networks. In Proceedings of the 2009 1st International Conference on Wireless Communication, Vehicular Technology, Information Theory and Aerospace Electronic Systems Technology, Aalborg, Denmark, 17-20 May 2009; pp. 813-817.

10. Cheng, M.; Ye, Q.; Cai, L. Cross-layer Schemes for Reducing Delay in Multihop Wireless Networks. IEEE Trans. Wirel. Commun. 2013, 12, 928-937. [CrossRef]

11. Merlin, C.J.; Heinzelman, W.B. Node Synchronization for Minimizing Delay and Energy Consumption in Low-power-listening MAC Protocols. In Proceedings of the 2008 5th IEEE International Conference on Mobile Ad Hoc and Sensor Systems, Atlanta, GA, USA, 29 September-2 October 2008; pp. 265-274.

12. Zhang, X.; Wang, C.; Tao, L. An Opportunistic Packet Forwarding for Energy-Harvesting Wireless Sensor Networks with Dynamic and Heterogeneous Duty Cycle. IEEE Sens. Lett. 2018, 2, 1-4. [CrossRef]

13. Luo, S.; Mao, X.; Sun, Y.; Ji, Y.; Tang, S. Delay Minimum Data Collection in the Low-duty-cycle Wireless Sensor Networks. In Proceedings of the 2012 IEEE Global Communications Conference (GLOBECOM), Anaheim, CA, USA, 3-7 December 2012; pp. 232-237.

14. Nazir, B.; Hasbullah, H.; Madani, S.A. Sleep/wake Scheduling Scheme for Minimizing End-to-end Delay in Multi-hop Wireless Sensor Networks. EURASIP J. Wirel. Commun. Netw. 2011, 2011, 92. [CrossRef]

15. Naveen, K.P.; Kumar, A. Relay Selection for Geographical Forwarding in Sleep-Wake Cycling Wireless Sensor Networks. IEEE Trans. Mob. Comput. 2013, 12, 475-488. [CrossRef]

16. Kim, J.; Lin, X.; Shroff, N.B.; Sinha, P. Minimizing Delay and Maximizing Lifetime for Wireless Sensor Networks with Anycast. IEEE/ACM Trans. Netw. 2010, 18, 515-528.

17. Cheng, L.; Niu, J.; Luo, C.; Shu, L.; Kong, L.; Zhao, Z.; Gu, Y. Towards Minimum-delay and Energy-efficient Flooding in Low-duty-cycle Wireless Sensor Networks. Comput. Netw. 2018, 134, 66-77. [CrossRef]

18. Liu, A.; Chen, Z.; Xiong, N.N. An Adaptive Virtual Relaying Set Scheme for Loss-and-delay Sensitive WSNs. Inf. Sci. 2018, 424, $118-136$. [CrossRef]

19. Wang, X.; Wu, X.; Zhang, X. Optimizing Opportunistic Routing in Asynchronous Wireless Sensor Networks. IEEE Commun. Lett. 2017, 21, 2302-2305. [CrossRef]

20. Yang, F.; Augé-Blum, I. Delivery Ratio-maximized Wakeup Scheduling for Ultra-low Duty-cycled WSNs Under Real-time Constraints. Comput. Netw. 2011, 55, 497-513. [CrossRef]

21. Fan, Z.; Bai, S.; Wang, S.; He, T. Delay-Bounded Transmission Power Control for Low-Duty-Cycle Sensor Networks. IEEE Trans. Wirel. Commun. 2015, 14, 3157-3170. [CrossRef] 
22. Niu, B.; Qi, H.; Li, K.; Liu, X.; Xue, W. Dynamic Scheming the Duty Cycle in the Opportunistic Routing Sensor Network. Concurr. Comput. Pract. Exp. 2017, 29. [CrossRef]

23. Wang, J.; Guan, L.; Wang, X.G.; Grigg, A.; Awan, I.; Phillips, I.; Chi, X. QoS Enhancements and Performance Analysis for Delay Sensitive Applications. In Proceedings of the 2009 International Conference on Advanced Information Networking and Applications, Bradford, UK, 26-29 May 2009; pp. 331-338.

24. Billingsley, P. Probability and Measure, 3rd ed.; Wiley Series in Probability and Mathematical Statistics; John Wiley \& Sons: New York, NY, USA, 1995; pp. 357-362, ISBN 0-471-00710-2.

25. Dao, T.N.; Yoon, S.; Kim, J. A Deadline-Aware Scheduling and Forwarding Scheme in Wireless Sensor Networks. Sensors 2016, 16, 59. [CrossRef] [PubMed]

26. Oliver, R.S.; Fohler, G. Probabilistic Estimation of End-to-end Path Latency in Wireless Sensor Networks. In Proceedings of the 2009 IEEE 6th International Conference on Mobile Adhoc and Sensor Systems, Macau, China, 12-15 October 2009; pp. 423-431.

27. Ye, W.; Heidemann, J.; Estrin, D. An Energy-efficient MAC Protocol for Wireless Sensor Networks. In Proceedings of the Twenty-First Annual Joint Conference of the IEEE Computer and Communications Societies, New York, NY, USA, 23-27 June 2002; Volume 3, pp. 1567-1576.

28. Gu, Y.; He, T. Dynamic Switching-Based Data Forwarding for Low-Duty-Cycle Wireless Sensor Networks. IEEE Trans. Mob. Comput. 2011, 10, 1741-1754. [CrossRef]

29. El-Hoiydi, A.; Decotignie, J.D., WiseMAC: An Ultra Low Power MAC Protocol for Multi-hop Wireless Sensor Networks. In Algorithmic Aspects of Wireless Sensor Networks: First International Workshop, Proceedings of the ALGOSENSORS 2004, Turku, Finland, 16 July 2004; Springer: Berlin/Heidelberg, Germany, 2004; pp. $18-31$.

30. Polastre, J.; Hill, J.; Culler, D. Versatile Low Power Media Access for Wireless Sensor Networks. In Proceedings of the 2nd International Conference on Embedded Networked Sensor Systems (SenSys '04), Baltimore, MD, USA, 3-5 November 2004; ACM: New York, NY, USA, 2004; pp. 95-107.

31. Buettner, M.; Yee, G.V.; Anderson, E.; Han, R. X-MAC: A Short Preamble MAC Protocol for Duty-cycled Wireless Sensor Networks. In Proceedings of the 4th International Conference on Embedded Networked Sensor Systems (SenSys '06), Boulder, CO, USA, 31 October-3 November 2006; ACM: New York, NY, USA, 2006; pp. 307-320.

32. Zorzi, M.; Rao, R.R. Geographic Random Forwarding (GeRaF) for Ad hoc and Sensor Networks: Energy and Latency Performance. IEEE Trans. Mob. Comput. 2003, 2, 349-365. [CrossRef]

33. Sun, Y.; Gurewitz, O.; Johnson, D.B. RI-MAC: A Receiver-initiated Asynchronous Duty Cycle MAC Protocol for Dynamic Traffic Loads in Wireless Sensor Networks. In Proceedings of the 6th ACM Conference on Embedded Network Sensor Systems (SenSys '08), Raleigh, NC, USA, 5-7 November 2008; ACM: New York, NY, USA, 2008; pp. 1-14.

34. University of California, Berkeley. Motescope Testbed in Soda Hall. Available online: https://www. millennium.berkeley.edu/wiki/sensornets (accessed on 10 June 2018).

35. Virtenio. Preon32 Wireless Module. Available online: https://www.virtenio.com/en/products/radiomodule.html (accessed on 10 June 2018).

36. Network Simulator ns-2. Available online: https://www.isi.edu/nsnam/ns/ (accessed on 11 May 2018).

37. McLachlan, G. Finite Mixture Models, 1st ed.; John Wiley \& Sons: New York, NY, USA, 2000; p. 6, ISBN 0-471-00626-2.

(C) 2018 by the authors. Licensee MDPI, Basel, Switzerland. This article is an open access article distributed under the terms and conditions of the Creative Commons Attribution (CC BY) license (http:/ / creativecommons.org/licenses/by/4.0/). 